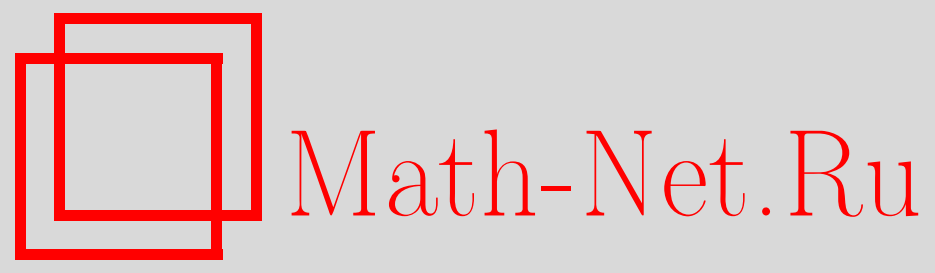

В. Н. Шевченко, Характеристические многочлены многоиндексных транспортных задач, Дискрет. матем., 2003, том 15, выпуск 2, 83-88

DOI: https://doi.org/10.4213/dm195

Использование Общероссийского математического портала Math-Net.Ru подразумевает, что вы прочитали и согласны с пользовательским соглашением http://www . mathnet.ru/rus/agreement

Параметры загрузки:

IP : 3.89 .185 .249

26 апреля 2023 г., 18:00:18 
Удк 519.10

\title{
Характеристические многочлены многоиндексных транспортных задач
}

\author{
(c) 2003 г. $\quad$ В. Н. Шевченко
}

Для матрицы $A^{T} A$, где $A$ - матрица ограничений многоиндексной транспортной задачи, найдены характеристический многочлен и базис из собственных векторов. Обсуждаются возможные применения полученных результатов.

1. Для исследования миноров $n$-го порядка $n \times m$ матрицы $A, n \leqslant m$, используется формула Бине-Коши (см., например, [1]), утверждающая, в частности, что

$$
s_{n}(A)=\operatorname{det} A A^{T}
$$

есть сумма квадратов всех миноров $n$-го порядка матрицы $A$. Если $s_{n}(A)$ удается вычислить (или хотя бы оценить), то этот прием позволяет оценить среднее значение квадрата минора матрицы $A$. Например, в [2] это сделано для матрицы, столбцами которой являются всевозможные векторы, содержащие $k$ единиц и $n-k$ нулей, и некоторых других матриц.

Разумеется, если ранг $r$ матрицы $A$ меньше $n$, то $s_{n}(A)=0$ и для получения нетривиальной информации о базисных минорах $r$-го порядка матрицы $A$ следует удалить линейно зависимые строки. Именно так Симмонард и Хедли нашли число базисов в транспортной задаче (см., например, [3]). Однако при этом может нарушиться структура задачи, за счет которой, собственно говоря, и удается найти $s_{n}(A)$. В такой ситуации может оказаться полезным характеристический многочлен матрицы $A A^{T}$

$$
\operatorname{det}\left(\lambda E-A A^{T}\right)=\sum_{j=0}^{n}(-1)^{j} s_{j}(A) \lambda^{n-j}
$$

Нетрудно видеть, что

$$
s_{j}(A)=\sum_{I, J}(\operatorname{det} A(I, J))^{2},
$$

где суммирование ведется по всем $j$-элементным подмножествам $I \subseteq\{1, \ldots, n\}$ и $J \subseteq\{1, \ldots, m\}$, а $A(I, J)$ - подматрица матрицы $A$ со строками из множества $I$ и столбцами из множества $J$.

Заметим, что иногда (например, в предлагаемой работе) удобнее использовать столь же информативный характеристический многочлен матрицы $A^{T} A$, связанный с (1) формулой

$$
\operatorname{det}\left(\lambda E-A^{T} A\right)=\lambda^{m-n} \operatorname{det}\left(\lambda E-A A^{T}\right) .
$$


Еще одно применение можно указать, если известен базис $Q=\left\{q_{1}, \ldots, q_{m}\right\}$ из собственных векторов матрищы $A^{T} A$. Так как условия $A^{T} A x=0$ и $A x=0$, очевидно, равносильны, то, выбрав из $Q$ все векторы, соответствующие нулевому собственному значению, получим базис пространства решений системы $A x=0$.

Следует отметить, что в теории графов успешно развивается целое направление, связанное с изучением характеристического многочлена матрищы смежности графа (см. монографию [4]).

В предлагаемой работе для матрицы $A=A\left(s ; n_{1}, \ldots, n_{k}\right)$ ограничений $k$-индексной $s$-валентной транспортной задачи найдены базис из собственных векторов, спектр (то есть набор собственных чисел и их кратностей) и характеристический многочлен $\Delta\left(\lambda ; s ; n_{1}, \ldots, n_{k}\right)$ матрицы $A^{T} A$.

2. Ограничения-равенства $k$-индексной $s$-валентной транспортной задачи (см., например, [3]) с неотрицательными переменными $x_{j_{1} \ldots j_{k}}, j_{\nu}=1, \ldots, n_{\nu}, v=1, \ldots, k$, можно записать в виде

$$
\sum_{j_{i_{1}}=1}^{n_{i_{1}}} \ldots \sum_{j_{i_{s}}=1}^{n_{i_{s}}} x_{j_{1} \ldots j_{k}}=b(J(I))
$$

где $J(I)=\left(j_{1}, \ldots, j_{i_{1}-1}, 0, j_{i_{1}+1}, \ldots, j_{i_{2}-1}, 0, j_{i_{2}+1}, \ldots, j_{i_{s}-1}, 0, j_{i_{s}+1}, \ldots, j_{k}\right)$ для всех $I=\left\{i_{1}, \ldots, i_{s}\right\}$ таких, что $1 \leqslant i_{1}<\ldots<i_{s} \leqslant k$ и всех $j_{\mu} \in\left\{1, \ldots, n_{\mu}\right\}$ при $\mu \notin I$. Таким образом, переменная $x_{j_{1}^{\prime} \ldots j_{k}^{\prime}}$ входит в уравнение, соответствующее $J(I)$, тогда и только тогда, когда $j_{v}^{\prime}=j_{v}$ для каждого $v \notin I$.

Положим $a\left(J(I), J^{\prime}\right)=1$, если $j_{v}^{\prime}=j_{v}$ для каждого $v \notin I$, и $a\left(J^{\prime}, J(I)\right)=0$ в противном случае. Тогда уравнение (3) можно переписать в виде

$$
\sum_{J^{\prime}} a\left(J(I), J^{\prime}\right) x_{J}^{\prime}=b(J(I))
$$

где суммирование ведется по всем $J^{\prime}=\left\{j_{1}^{\prime}, \ldots, j_{k}^{\prime}\right\}$ таким, что $j_{\nu}^{\prime} \in\left\{1, \ldots, n_{\nu}\right\}$, $v=1, \ldots, k$.

Будем говорить, что набор $J^{\prime}=\left\{j_{1}^{\prime}, \ldots, j_{k}^{\prime}\right\}$ лексикографически предшествует набору $J^{\prime \prime}=\left\{j_{1}^{\prime \prime}, \ldots, j_{k}^{\prime \prime}\right\}$ (в этом случае пишем $J^{\prime} \prec J^{\prime \prime}$ ), если найдется такое $v \in\{1, \ldots, k\}$, при котором $j_{1}^{\prime}=j_{1}^{\prime \prime}, \ldots, j_{v-1}^{\prime}=j_{v-1}^{\prime \prime}$ и $j_{v}^{\prime}<j_{v}^{\prime \prime}$. Это отношение линейно упорядочивает индексы $J^{\prime}$ переменных и $J(I)$ уравнений. Ясно, в частности, что если $I^{\prime} \prec I^{\prime \prime}$, то $J^{\prime}\left(I^{\prime}\right) \prec J^{\prime \prime}\left(I^{\prime \prime}\right)$.

Обозначим через $A\left(s ; n_{1}, \ldots, n_{k}\right)$ матрицу, составленную из элементов $a\left(J^{\prime}, J(I)\right)$ при лексикографическом упорядочивании наборов индексов. Зафиксировав $I$, рассмотрим ее подматрицу $A\left(I ; n_{1}, \ldots, n_{k}\right)$ со строками из всевозможных $J(I)$. Для ее описания удобно воспользоваться кронекеровым произведением матриц (определение и свойства см., например, в [1]). Напомним, что кронекеровым произведением $A \times B$ прямоугольных матриц $A$ и $B$ размеров $n \times m$ и $p \times q$ соответственно называется матрица $C$ размера $(n p, m q)$ следующего блочного строения:

$$
C=\left(\begin{array}{cccc}
a_{11} B & a_{12} B & \ldots & a_{1 m} B \\
a_{21} B & a_{22} B & \ldots & a_{2 m} B \\
& & \ldots & \\
a_{n 1} B & a_{n 2} B & \ldots & a_{n m} B
\end{array}\right)
$$


Обозначим через $I(m, n)$ матрицу размера $m \times n$, каждый элемент которой равен 1 , а через $E_{n}$ - единичную матрицу $n$-го порядка.

Лемма 1. Для любых $s=|I| u k$ таких, что $0 \leqslant s \leqslant k$, справедливо равенство

$$
A\left(I ; n_{1}, \ldots, n_{k}\right)=A_{I}\left(1, n_{1}\right) \times \ldots \times A_{I}\left(k, n_{k}\right),
$$

где $A_{I}\left(v, n_{v}\right)=I\left(1, n_{v}\right) n p u v \in I u A_{I}\left(v, n_{v}\right)=E_{n_{v}}$ npu $v \notin I$.

Доказательство. Доказательство проведем индукцией по $k$. При $k=1$ утверждение очевидно, так как для $s=0$ система (3) превращается в систему $x_{j_{1}}=b_{j_{1}}, j_{1}=1, \ldots, n_{1}$, а для $s=1$ получаем одно уравнение

$$
\sum_{j_{1}=1}^{n_{1}} x_{j_{1}}=b_{0} .
$$

Предположив, что утверждение доказано для $k-1$, докажем его для $k$. Если $k \in I$, то $a\left(J(I), J^{\prime}\right)=1$ при $j_{k}^{\prime} \in\left\{1, \ldots, n_{k}\right\}$, следовательно,

$$
A\left(I ; n_{1}, \ldots, n_{k}\right)=A\left(I ; n_{1}, \ldots, n_{k-1}\right) \times I\left(1, n_{k}\right) .
$$

Если же $k \notin I$, то $a\left(J(I), J^{\prime}\right)=0$ при $j_{k}^{\prime} \neq j_{k}$ и, следовательно,

$$
A\left(I ; n_{1}, \ldots, n_{k}\right)=A\left(I ; n_{1}, \ldots, n_{k-1}\right) \times E_{n_{k}} .
$$

Лемма 2. Если $A=A\left(I ; n_{1}, \ldots, n_{k}\right)$, mo

$$
A^{T} A=B_{I}\left(1, n_{1}\right) \times \cdots \times B_{I}\left(k, n_{k}\right),
$$

где $B_{I}\left(v, n_{v}\right)=I\left(n_{v}, n_{v}\right)$ npu $v \in I u B_{I}\left(v, n_{v}\right)=E_{n_{\nu}}$ npu $v \notin I$.

Доказательство следует из формулы (5) и следующих свойств кронекерова произведения: $(A \times B)(C \times D)=A C \times B D$ и $(A \times B)^{T}=A^{T} \times B^{T}$.

Пусть

$$
Q_{n}=\left(\begin{array}{cccc}
1 & 1 & \ldots & 1 \\
1 & -1 & \ldots & 0 \\
& & \ldots & \\
1 & 0 & \ldots & -1
\end{array}\right)
$$

- матрица $n$-го порядка и $q_{j}$ - ее $j$-й столбец. Нетрудно проверить, что

$$
I(n, n) q_{j}=\lambda_{j} q_{j}
$$

где $\lambda_{j}=n$ при $j=1$ и $\lambda_{j}=0$ при $j=2, \ldots, n$. Так как $\operatorname{det} Q_{n}=(-1)^{n+1} n$, столбцы матрищы $Q_{n}$ составляют базис из собственных векторов матрицы $I(n, n)$. Разумеется, собственные векторы можно выбирать и по-другому, однако предложенный способ дает целочисленную и симметричную матрицу $Q_{n}$.

Лемма 3. Столбчы матрицы $Q=Q_{n_{1}} \times \cdots \times Q_{n_{k}}$ составляют базис из собственных векторов матрицы $A^{T} A$, где $A=A\left(I ; n_{1}, \ldots, n_{k}\right)$. 
Доказательство. Для доказательства леммы рассмотрим при любом $J=\left\{j_{1}, \ldots, j_{k}\right\}$ столбец $q_{J}=q_{j_{1}} \times \cdots \times q_{j_{k}}$ матрицы $Q$ и покажем, что $A^{T} A q_{J}=\mu_{J}^{I} q_{J}$. Из формулы (6) следует, что

$$
A^{T} A q_{J}=\left(B_{l}\left(1, n_{1}\right) q_{j_{1}}\right) \times \cdots \times\left(B\left(k, n_{k}\right) q_{j_{k}}\right) .
$$

Если найдется $\nu \in I$ такое, что $j_{\nu}>1$, то

$$
B_{I}\left(\nu, n_{\nu}\right) q_{j_{v}}=I\left(n_{\nu}, n_{\nu}\right) q_{j_{\nu}}=0,
$$

и следовательно, $\mu_{J}^{I}=0$. В противном случае $B_{I}\left(\nu, n_{\nu}\right) q_{j_{\nu}}=n_{\nu} q_{\nu}$ при $\nu \in I$ и $B_{I}\left(\nu, n_{v}\right) q_{j_{v}}=q_{j_{v}}$ при $\nu \notin I$, следовательно, $A^{T} A q_{J}=\mu_{J}^{I} q_{J}$, где

$$
\mu_{J}^{I}=\prod_{i \in I} n_{j_{i}}
$$

Теорема 1. Если $A=A\left(s ; n_{1}, \ldots, n_{k}\right)$-матрица $k$-индексной s-валентной транспортной задачи, то столбцы матрицы $Q=Q_{n_{1}} \times \cdots \times Q_{n_{k}}$ составляют базис из собственных векторов матрицы $A^{T} A$.

Для любого $J=\left\{j_{1}, \ldots, j_{k}\right\}$ собственное число $\mu_{J}$, соответствующее столбиу $q_{J}=q_{j_{1}} \times \cdots \times q_{j_{k}}$, вычисляется по формуле

$$
\mu_{J}=\sigma_{s}\left(\lambda\left(1, j_{1}\right), \ldots, \lambda\left(k, j_{k}\right)\right)
$$

где

$$
\sigma_{s}\left(\alpha_{1}, \ldots, \alpha_{k}\right)=\sum_{1 \leqslant i_{1}<\cdots<i_{s} \leqslant k} \alpha_{i_{1}} \alpha_{i_{2}} \ldots \alpha_{i_{s}}
$$

- $s$-я элементарная симметрическая функиия от $\alpha_{1}, \ldots, \alpha_{k}, \sigma_{0}\left(\alpha_{1}, \ldots, \alpha_{k}\right)=1$, и для $\nu=1, \ldots, k$

$$
\lambda\left(\nu, i_{\nu}\right)= \begin{cases}n_{\nu} & n p u j_{\nu}=1, \\ 0 & n p u j_{\nu}=2, \ldots, n_{\nu} .\end{cases}
$$

Доказательство. Для доказательства заметим, во-первых, что

$$
A^{T} A=\sum_{I} A^{T}\left(I ; n_{1}, \ldots, n_{k}\right) A\left(I ; n_{1}, \ldots, n_{k}\right),
$$

где суммирование ведется по всем $s$-элементным подмножествам множества $\{1, \ldots, k\}$. Отсюда и из леммы 2 следует, что

$$
A^{T} A=\sum_{I} B_{I}\left(1, n_{1}\right) \times \ldots \times B_{I}\left(k, n_{k}\right) .
$$

Воспользовавшись леммой 3 , получаем доказательство первого утверждения, так как

$$
A^{T} A q_{J}=\sum_{I} \mu_{J}^{I} q_{J}
$$

Используя полученное в лемме 3 выражение для $\mu_{J}^{I}$, нетрудно проверить, что

$$
\mu_{J}^{I}=\prod_{i \in I} \lambda\left(i, j_{i}\right)
$$

откуда следует второе утверждение теоремы. 
Следствие 1. Базис пространства решений системы линейных уравнений

$$
A\left(s ; n_{1}, \ldots, n_{k}\right) x=0
$$

состоит из столбцов матричы $Q=Q_{n_{1}} \times \cdots \times Q_{n_{k}}$, соответствующих наборам $J=\left\{j_{1}, \ldots, j_{k}\right\}$, содержачим не более $s-1$ единич. Их число $t=t\left(s ; n_{1}, \ldots, n_{k}\right)$ равно

$$
\sum_{i=0}^{s-1} \sigma_{k-i}\left(n_{1}-1, \ldots, n_{k}-1\right)=\prod_{j=1}^{k} n_{j}-\sum_{i=s}^{k} \sigma_{k-i}\left(n_{1}-1, \ldots, n_{k}-1\right) .
$$

В частности, для планарной задачи

$$
t\left(1 ; n_{1}, \ldots, n_{k}\right)=\prod_{j=1}^{k}\left(n_{j}-1\right)
$$

а для аксиальной

$$
t\left(k-1 ; n_{1}, \ldots, n_{k}\right)=\prod_{j=1}^{k} n_{j}-\sum_{j=1}^{k} n_{j}+k-1 .
$$

Следствие 2. Пусть $M \subseteq\{1, \ldots, k\},|M| \geqslant s$. Тогда

$$
\sigma_{s}^{M}=\sum_{I \subseteq M} \prod_{j \in I} n_{j}
$$

¿е суммирование ведется по всем $s$-элементным подмножествам I множества $M$, является ненулевым собственным значением кратности

$$
\prod_{j \notin M}\left(n_{j}-1\right)
$$

и других ненулевых собственных значений нет.

Полученные результаты позволяют находить характеристический многочлен $\Delta\left(\lambda ; s ; n_{1}, \ldots, n_{k}\right)$. Например, для аксиальной задачи

$$
\Delta\left(\lambda ; k-1 ; n_{1}, \ldots, n_{k}\right)=\lambda^{t\left(k-1 ; n_{1}, \ldots, n_{k}\right)}\left(\lambda-\sum_{i=1}^{k} \prod_{j \neq i} n_{j}\right) \prod_{i=1}^{k}\left(\lambda-\prod_{j \neq i} n_{j}\right)^{\left(n_{i}-1\right)}
$$

Дальше для упрощения формул будем считать, что $n_{1}=\cdots=n_{k}=n$. Положим $\Delta(\lambda ; s, k, n)=\Delta\left(\lambda ; s ; n_{1}, \ldots, n_{k}\right)$, если $n_{1}=\cdots=n_{k}=n$.

Следствие 3. Справедливо равенство

$$
\Delta(\lambda ; s, k, n)=\lambda^{\sum_{i=0}^{s-1}\left(\begin{array}{l}
k \\
i
\end{array}\right)(n-1)^{k-i}} \prod_{j=s}^{k}\left(\lambda-\left(\begin{array}{l}
j \\
s
\end{array}\right) n^{s}\right)^{\left(\begin{array}{l}
k \\
j
\end{array}\right)(n-1)^{k-j}}
$$


Из (9) для аксиальных задач следует, что

$$
\Delta(\lambda ; k-1, k, n)=\lambda^{n^{k}-k n+k-1}\left(\lambda-k n^{k-1}\right)\left(\lambda-n^{k-1}\right)^{k(n-1)}
$$

и, значит, сумма квадратов ранговых миноров (порядок которых равен рангу $r$ матрицы $A$, в данном случае $r=k(n-1)+1)$ равна $k n^{(k-1)(k(n-1)+1)}$. Отсюда нетрудно получить, что при $k \geqslant 3$ средняя величина квадратов рассматриваемых миноров с ростом $n$ стремится к бесконечности с экспоненциальной скоростью. Этот результат получен в кандидатской диссертации А. П. Ильичева [5] другим способом.

Путь, предлагаемый здесь, позволит получать результаты для произвольного $s$ и для миноров меньшего, чем $r$ порядка.

\section{Список литературы}

1. Воеводин В. В., Кузнецов Ю. А., Матрищы и вычисления. Наука, Москва, 1984.

2. Шевченко В. Н., Качественные вопросы челочисленного программирования. Наука, Москва, 1995.

3. Емеличев В. А., Ковалев М. М., Кравцов М. К., Многогранники, графы, оптииизаџия. Наука, Москва, 1981.

4. Цветкович Д., Дуб М., Захс Х., Спектры графов. Теория и применение. Наукова думка, Киев, 1984.

5. Ильичев А. П., Исследование многогранников многоиндексных транспортных задач. Диссерт. на соискание степени канд. физ.-мат. наук. Горький, 1988.

Статья поступила 11.02.2002. 